\title{
PRO-VOCATION MARIONNETTES Les Rencontres Internationales sur la Formation aux Arts de la Marionnette PRO-VOCAÇÃO MARIONETES
} Encontros Internacionais de Formação nas Artes da Marionete

Irina Niculescu ${ }^{1}$ 


\section{Résumé}

Dans ce texte, la marionnettiste Irina Niculescu rapporte les détails et les détails des deux Rencontres Internationales de Formation aux Arts de la Marionnette. Survenu en 2015 en France et en 2017 en Roumanie. Enfin, la réunion de 2019 aura lieu au Brésil, à l'UDESC-SC.

Mon-C: Entraînement aux arts de la marionnette Réunions internationales de formation des élèves; Étudiants en arts marionnettes

\section{Resumo}

Neste texto a marionetista Irina $\mathrm{Ni}$ culescu relata os encaminhamentos e detalhes dos dois Encontro Internacional de Formação nas Artes da Marionete. Ocorridos em 2015 na França e em 2017 na Romênia. E destaca, por fim, que o encontro de 2019 será realizado no Brasil, na UDESC - SC.

Palavras-chave: Formação nas artes da Marionete; Encontros Internacionais de Formação da Marionetista; Estudiosos das artes da Marionete 


\section{Le contexte théâtral. Mes racines}

Je suis metteur en scène. Je raconte des histoires qui parlent de l'âme humaine. Mes spectacles expriment et partagent avec le public mes réflexions sur la vie, ma joie, mes questionnements, mes craintes et mes utopies. Pour cela, je m'accompagne de poètes, d'écrivains, de peintres et de sculpteurs. La marionnette a pour moi une essence tragi-comique, car elle est toujours liée aux mains visibles ou invisibles du manipulateur. Elle est la métaphore de la condition humaine. L'acte de la manipulation, ainsi que l'interdépendance du marionnettiste et de la marionnette contribuent à ce double effet d'innocence et d'irrévérence que la marionnette exerce sur nous avec tant de charme. La marionnette a un extraordinaire pouvoir d'évocation. Elle existe à la frontière fragile entre l'animé et l'inanimé. Elle nous entraîne dans une fiction qui devient plus réelle que la réalité.

Créer, enseigner. Jeune pédagogue, j'ai commencé à enseigner la mise en scène et le jeu avec la marionnette. J'ai développé ma propre technique pour enseigner la marionnette, visant à atteindre l'excellence en interprétation et j'ai exploré en profondeur l'essence des différents types de marionnettes et de leur dramaturgie spécifique. J'ai cherché à découvrir ce qui fait le charme de la marionnette, son mystère et sa force, et quelle est la relation entre chaque genre de marionnette et son territoire dramatique privilégié. Mon aventure théâtrale a commencé au milieu des années '70.

Je fais partie de la génération des créateurs qui ont amené la marionnette à sortir du castelet, découvrir l'espace ouvert et rencontrer le marionnettiste. Ce changement a été essentiel dans le renouveau du théâtre; il a généré une énergie créatrice exubérante. Une autre conception de l'espace et par la suite de l'éclairage, un autre regard sur le rapport des proportions. La matière brute et les matériaux de construction ont été redécouverts et choisis pour leur potentiel dramatique. La relation entre la marionnette et le marionnettiste est devenue porteuse de nouveaux sens qu'il faudra définir. Les artistes ont commencé à explorer de nouvelles approches théâtrales à partir d'objets, de matières et de formes inusitées.

Ce souffle créateur a stimulé l'imaginaire et enrichi la palette des moyens d'expression. Une autre approche de l'écriture scénique, un autre regard sur la mise en scène multiplient les formes dramaturgiques.

Le public se diversifie.

\section{Le statut du marionnettiste}

Pour faire face aux nouvelles exigences nous avons changé le statut du marionnettiste qui est devenu un artiste de la scène ouverte maîtrisant de multiples habiletés et savoirs tels le jeu d'acteur, la danse et la musique, une variété de techniques traditionnelles ainsi que des capacités à inventer de nouvelles marionnettes et de nouvelles techniques et technologie. Une technique de formation professionnelle unique n'a vraiment jamais existé. 


\section{Le statut de la marionnette}

Au milieu de ce tumulte la marionnette subit aussi de nombreux changements de statut et d'apparence. Elle passe de l'objet figuratif aux objets d'inspiration utilitaire, aux matières diverses choisit pour leurs qualités théâtrales, leur apparence et leur vibration, aux masques corporels et aux formes animées.

En 2008, à ma première rencontre avec les étudiants du Programme de formation en théâtre de marionnettes contemporain de l'Université du Québec a Montreal (DESS), une étudiante m'a demandé « qu'est-ce que c'est « la marionnette » de nos jours ? ». Question essentielle, car elle pointe vers la multitude des formes contemporaines, produites par une inventivité débordante, presque obsessive qui oublie parfois la qualité de métaphore de la marionnette et la dérobe de sa substance. Surprise par la pertinence de cette question et considérant qu'une définition unique n'ai pas possible j'ai pu lui répondre en exprimant ce que la marionnette est son théâtre signifient pour moi.

\section{La formation professionnelle}

La diversité des concepts artistiques rend nécessaire une diversité de modalités de formation: programmes et diplômes universitaires, écoles de théâtre indépendantes, ateliers, classes de maîtres et formation au sein des compagnies de théâtre. Chaque type de formation fait résonner une vision particulière du théâtre, une certaine conception de la « marionnette», du «théâtre de marionnettes» et correspond à une certaine philosophie de l'éducation.

Quels sont les objectifs de ces types de programme de formation ? À qui enseignons-nous ? Et à qui voulons-nous enseigner ? Quels sont les critères d'admission ? Quels sont les défis des formations actuelles; nos succès et nos échecs, nos doutes, nos questionnements ? Comment définissons-nous ce qu'est la marionnette et ce qu'est le théâtre de marionnettes aujourd'hui ? Quel genre de formation envisageons-nous pour l'avenir? Car les nouveaux

[...] langages qui - pour ce qu'ils disent du théâtre de marionnettes - parle des limites fluctuantes et pour cette raison aujourd'hui presque indéfinissables de l'art de la marionnette, de la rencontre qui s'accomplit actuellement entre les différents arts sur la scène du théâtre de marionnettes, de la responsabilité que prennent les jeunes artistes pour leur représentation, de la conscience avec laquelle ils décrivent et interroge leur réalité sociale, de la rigueur avec laquelle ils mettent en question la tradition, de la luminosité du puzzle qu'ils composent avec des modes de représentation traditionnels et nouvellement découverts, de l'évidence avec laquelle ils exigent une formation moderne et l'espace nécessaire pour un travail de création. (Silvia Brendenal)

\section{La première Rencontre internationale sur la formation aux arts de la marionnette Du 16 au 18 septembre 2015. Institut Internationale de la Marionnettes, Charleville-Mézières, France}

Pour répondre à ces questions et à bien d'autres, en 2013, en complicité avec des collègues de la Commission pour la Formation Professionnelle de l'Union In- 
ternationale de la Marionnette (UNIMA) je propose que la commission organise une rencontre internationale sur la formation aux arts de la marionnette. A ce moment la Commission considère que nous ne sommes pas suffisamment prêts pour assumer un tel projet. Nous reprenons la proposition en 2014 , lors de la réunion de la Commission à Matanzas, à Cuba et cette fois-ci la Commission embrasse l'idée. Nous commençons à réfléchir à la thématique et à l'organisation de la rencontre.

La Première Rencontre Internationale sur la Formation aux Arts de la Marionnette aura lieu a Charleville-Mézières, en France et sera accueillie par l'Institut Internationale de la Marionnette. Elle rassemblera artistes, pédagogues et chercheurs représentant les diverses modalités de formation, réunis pour en questionner les bases, définir ensemble ce que sont «la marionnette» et «le théâtre de marionnettes» aujourd'hui, réfléchir sur la finalité des formations (quel type de marionnettiste veut-on former?), leurs contenus (quelles compétences cherche t-on à transmettre ?), et revoir les différentes modalités et philosophies d'enseignement. Partager les défis de la formation dans le contexte actuel, les succès, les échecs, les questionnements, pour bâtir des ponts solides entre les artistes, les pédagogues et les différentes structures de formation.

La Rencontre réunit 50 invités venus de 23 pays : France, Italie, Espagne, Allemagne, Grande Bretagne, Bulgarie, Roumanie, Hongrie, Norvège, République Tchèque, Pologne, Slovaquie, Russie, Suisse, Inde, Chine, Sénégal, Brésil, Argentine, Cuba, Israël, États Unis, Canada; à peu près 70 personnes assistent à l'une ou l'autre des conférences.

\section{Les thèmes présentés}

- Les secrets ontologiques de la marionnette - Henryk Jurkowski

- Philosophies de la formation et visions pédagogiques - Marek Waszkiel

- Un théâtre des marionnettistes - Pierre Blaise

- Quel acteur marionnettiste pour demain? - Éloi Recoing

- Défis, succès, échecs - Julika Mayer, Dinaïg Stall, Kata Csato

- Réflexions sur les structures et les programmes de formation en milieu universitaire - Tito Lorefice, Bart Roccerberton, Marthe Adam

- La problématique de la mise en scène et de la dramaturgie dans la formation en théâtre de marionnettes - Irina Niculescu

- De l'importance d'apprendre les techniques de fabrication de marionnettes Greta Bruggeman

- Les arteils du marionnettiste - la marionnette et le corps de l'acteur - Claire Heggen

- Enseigner la marionnette contemporaine sous ses différentes formes dans une formation traditionnelle. Enseigner les différents types de marionnettes traditionnelles dans une formation contemporaine - Anurupa Roy, Xiaoxin Wang.

- La marionnette et le théâtre alternatif à l'École de Théâtre Alternatif (DAMU), Prague, République Tchèque - Jiri Adamek

- Des formes traditionnelles aux formes actuelles - Fabrizio Montecchi, Joan Baixas 
- La Rencontre se termine par un regard de synthèse préparée par Laure Garcin-Marrou, maître de conférence en Études Théâtrales et Visuelles de l'Université de Toulouse Jean Jaurés, en France.

Les participants pratiquent des approches pédagogiques qui diffèrent selon leur culture, leur vision du théâtre et leur philosophie de l'enseignement, les buts de la formation et selon les lieux où ils travaillent, selon les défis auxquels ils font face, et selon la génération auxquelles ils appartiennent. Metteurs en scène, dramaturges, artistes en arts visuels, scénographes et concepteurs, mimes corporelles et spécialistes du mouvement, théoriciens du théâtre ou chercheurs et directeurs de programmes d'études en théâtre de marionnettes, d'objets ou théâtre d'ombre.

La plupart des conférenciers abordent la problématique du choix des processus pédagogiques. Leurs expériences et points de vue divergent. Certains, pensent qu'il faut placer l'étudiant au centre de l'enseignement et partir de ses besoins, tandis que d'autres affirment qu'il faut assumer d'abord l'apprentissage des genres traditionnels et ensuite seulement aborder les nouvelles formes de marionnettes.

Les questions posées dès le début de la Rencontre Internationale «Qu'est-ce qu'une marionnette? », "Qu'est-ce que le théâtre de marionnettes contemporain » ont été abordée par plusieurs conférenciers. Nous avons retenu qu'il n'y a pas une réponse unique, qui définisse la marionnette et son théâtre. " Théâtre de marionnettes », " théâtre de figures », " théâtre des formes animées », " théâtre d'animation » sont parmi les noms qui essayent de contenir la diversité des formes théâtrales contemporaines dans l'univers du théâtre de marionnettes.

L'un des buts de la Rencontre était de mettre en contact les différentes philosophies et structures d'enseignement et d'explorer les possibilités de créer des ponts entre les structures universitaires et les structures alternatives; lors de l'événement, des contacts professionnels entre les participants se sont établis en vue de futurs échanges.

La Rencontre Internationale a été intense et dynamique et elle s'est déroulée dans une atmosphère d'enthousiasme. Nous n'avons certainement pas réussi à approfondir les sujets proposés, car les participants étaient curieux de se connaître et de se raconter. Mais nous avons provoqué la naissance d'une communauté de réflexion et d'échanges sur le théâtre de marionnettes contemporain et les diverses approches créatrice et pédagogiques qui le définissent aujourd'hui.

Suite à la demande unanime des participants, la Commission pour la Formation Professionnelles de l'UNIMA a décidé d'organiser une série de rencontres internationales biannuelles sur la formation aux arts de la marionnette, ceci en collaboration avec des écoles universitaires de théâtre, des laboratoires de formation et de création et des Centres Nationales de l'UNIMA.

Chose importante: ces rencontres réuniront théorie et pratique et auront lieu dans différentes régions culturelles du monde. 


\section{La Deuxième Rencontre internationale sur la formation aux arts de la ma- rionnette Du 20 au 24 juin 2017. \\ Théâtre Municipal Tony Bulandra, Targoviste, Roumanie \\ Avec la collaboration de l'UNIMA Roumanie}

La deuxième Rencontre Internationale sur la Formation aux Arts de la Marionnette propose de reprendre et d'approfondir les transformations esthétiques profondes qu'a vécu le théâtre de marionnettes depuis les années 70: l'évolution du statut des marionnettistes, l'évolution conceptuelle et poétique des espaces scéniques, les nouvelles formes du théâtre de marionnettes et aussi l'art en tant qu'outil pédagogique, l'art et la société, les différents lieux de formation, formels et informels : lieux alternatifs, lieux institutionnels et leur apport spécifique.

Le matin auront lieu deux ateliers pratiques d'une heure et demi chacun dirigés par des artistes avec des approches pédagogiques personnels et unique venant du domaine de la danse, de la marionnette et des arts digitales. France, de Belgique, de l'Iran et du Brésil. Les soirées seront animées par des rencontres amicales et la présentation des diverses compagnies de théâtre.

\section{Les thèmes présentés}

1. La formation aux arts de la marionnette dans le cadre de la compagnie et du laboratoire théâtral Arketal - Greta Bruggeman, France

2. La formation aux arts de la marionnette dans le cadre de la compagnie KatKatha - Anurupa Roy, Inde

3. Les transformations de la marionnette - Katerina Dolenska, République Tchèque

4. Être ou ne pas être un marionnettiste professionnel - Anca Doina Ciobotaru, Roumanie

5. Dialogue entre tradition et innovation dans la pédagogie actuelle - Adriana Schneider, Brésil. Ranjana Pandey, Inde. Izabela Brochado, Brésil. Cariad Astles, Grande Bretagne.

6. Approches interdisciplinaires dans la mise en scène au théâtre de marionnettes et liens avec la pédagogie - Fabrizio Montecchi, Italie. Nicole Mossoux, Belgique, Shiva Massoudi, Iran. Anne Helgesen, Norvege. Irina Niculescu, Etats Unis.

7. Les systèmes pédagogiques selon les besoins des élèves - Lucile Bodson

8. La formation aux arts de la marionnette en Turquie - Isinsu Ersan, Turquie

9. La formation aux arts de la marionnette à l'Université des arts théâtraux et cinématographiques et à l'étranger - Ana Craciun-Lambru, Roumanie

10. Les rôles de l'objet animé du corps et de l'espace dans le théâtre de marionnette actuel - Mario Piragibe, Brésil

11. Mimésis complexe et complexité des figures dans le théâtre contemporain Cristina Grazioli, Italie et Didier Plassard, France.

12. Comment implanter la marionnette dans le cœur de quelqu'un - les défis d'enseigner la marionnette à l'université - Miguel Vellinho, Brésil

13. Les expériences et les productions artistiques comme découverte des com- 
pétences personnelles et leurs et leur amélioration au niveau technique, éthique et humain - Philippe Choulet, France

14. La présence de la mise en scène dans la formation théâtrale aux arts de la marionnette - Kata Czato, Hongrie

15. Réflexions sur les trois jours de travail - Marek Waszkiel, Pologne.

\section{En guise de conclusion}

La deuxième rencontre sur la formation a réunis autour de 60 participants venant de Brésil, Argentine, Turquie, Singapore, Bulgarie, Hongrie, Norvège, Italie, France, Pologne, Argentine, Israël, Slovénie, États-Unis, Canada, Grande-Bretagne. Autour de 400 observateurs ont suivis les présentations et les tables rondes sur internet live. La présence des étudiants de l'Université des Arts de lasi (Roumanie) et leur participation aux ateliers cote a cote avec des artistes expérimentés, leur désir d'apprendre et leur enthousiasme ont donné a la rencontre un coté pratique et concret. Le dialogue entre les artistes, les pédagogues, les chercheurs et les philosophes présents ont apporté des angles de vue différents, provoquants et enrichissants.

Nous ne sommes pas prêts à définir des conclusions durables. Une chose est sure : nous sommes tous d'accord que le théâtre s'apprend. L'apprentissage prend des voies différentes, l'enseignement aussi, mais nous sommes tous d'accord que l'enseignement doit évoluer en suivant les nouvelles exigences du théâtre de marionnettes et de formes animées qui est en permanente recherche et transformation.

Les questions demeurent et accompagnent toujours le créateur et l'enseignant. Ce qui a provoqué surprise et malaise quelques années auparavant, aujourd'hui fait la norme. Mais la communication et l'échange sont établis,

L'accueil chaleureux du Théâtre Tony Bulandra et de son équipe technique et administratif, leur présence personnalisée prête a résoudre tous les problèmes d'organisation, la collaboration de l'UNIMA Roumanie ont fait que les trois jours et demi de travail, surchargés d'ateliers pratiques, de présentations et de tables-rondes se déroulent dans une atmosphère intense mais agréable. Les diners tard dans la soirée dans les petits restaurants de la ville, les petits déjeuners sur la terrasse de l'hôtel ont créée l'ambiance pour que des affinités se rencontrent et des amitiés naissent. Le programme de la rencontre a fini avec la visite de l'ancienne ville médiévale de Targoviste entourée par des monastères du 13 eme et 14 siècle. De retour au théâtre nous avons conclu la rencontre avec une fête qui c'est éteint tard dans la nuit.

\section{La Troisième Rencontre internationale sur la formation aux arts de la ma- rionnette Du 15 au 20 mai 2019. \\ Université Nationale de Santa Catarina, Florianópolis, Brésil}

\section{Préambule}

La troisième rencontre abordera « la problématique de l'enseignement de la mise en scène liée à la diversité des processus de création du spectacle ».

La mise en scène et la dramaturgie sont peut-être les plus grands défis du théâ- 
tre de marionnettes contemporain, de la performance, de l'installation. Quelque soit la forme de théâtre ou d'évènement théâtral que nous créons, il faut que cela raconte l'humain dans sa quête existentielle. Car inspirés par le potentiel de ces nouveaux moyens expressifs, des artistes venant du monde de la danse, du mime, des arts plastiques se sont orientés vers la marionnette et cette rencontre a fait naitre de nombreuses formes inédites et spectaculaires, qui conjuguent la marionnette avec le corps de l'acteur, qui invente le masque porté et le corps fictif, et qui converge à l'intersection du jeu de la marionnette avec celui de l'acteur, du danseur et du mime corporel.

\section{L'imaginaire envahit le plateau}

La mise en scène change de statut, elle devient un acte conceptuel, le metteur en scène un créateur.

Quelle dramaturgie faut-il inventer pour un théâtre qui cherche en permanence sa forme et qui se modifie continuellement ? Quels seront les repères de l'écrivain, du dramaturge, du metteur en scène ? Faut-il créer des règles et des abécédaires ? Serait-il même possible? Nous savons bien qu'il n'y a pas de vérité unique, ni des vérités définitives. Le théâtre contemporain part plus rarement d'un texte écrit pour la scène. Ses sources sont souvent un texte littéraire, une pièce musicale, un thème, une idée, une envie de questionner la réalité, une envie d'avoir un impact social.

La base dramaturgique est créée pour le spectacle ; l'écriture et la mise en scène doivent travailler dans une relation de complicité, évoluant ensemble jusqu'à la fin du processus de création. Est-ce que la mise en scène s'enseigne ? Pourquoi si peu d'écoles universitaires et alternatives offrent des cours et des stages de mise en scène ? Est-ce que créer des mises en scène peut s'apprendre seulement par l'expérience ? Pleins de questions que nous allons aborder, en dialoguant avec des créateurs qui ont inventé des processus de création personnelles et uniques et des metteurs en scènes pédagogues, en parcourant a grandes enjambées l'histoire de la mise en scène et de ses défis et en pénétrant dans les secrets de la création du théâtre de marionnettes et de formes animées contemporain.

J'aimerais terminer avec quelques amuse-gueules en citant les surnoms que le metteur en scène a reçu au long du temps: jardinier des esprits, docteur des sensations, accoucheur du non-dit, cordonnier des situations, cuisinier des discours, roi du théâtre et servant du plateau, jongleur et magicien, pierre de touche du public, diplomate, économiste, infirmier, chef d'orchestre, interprète (traducteur), peintre et costumier...

Irina Niculescu - décembre 2017

Ce texte est également publié en portugais dans cette édition.

Reçu: 10/07/2018

Approuvé: 10/07/2018 


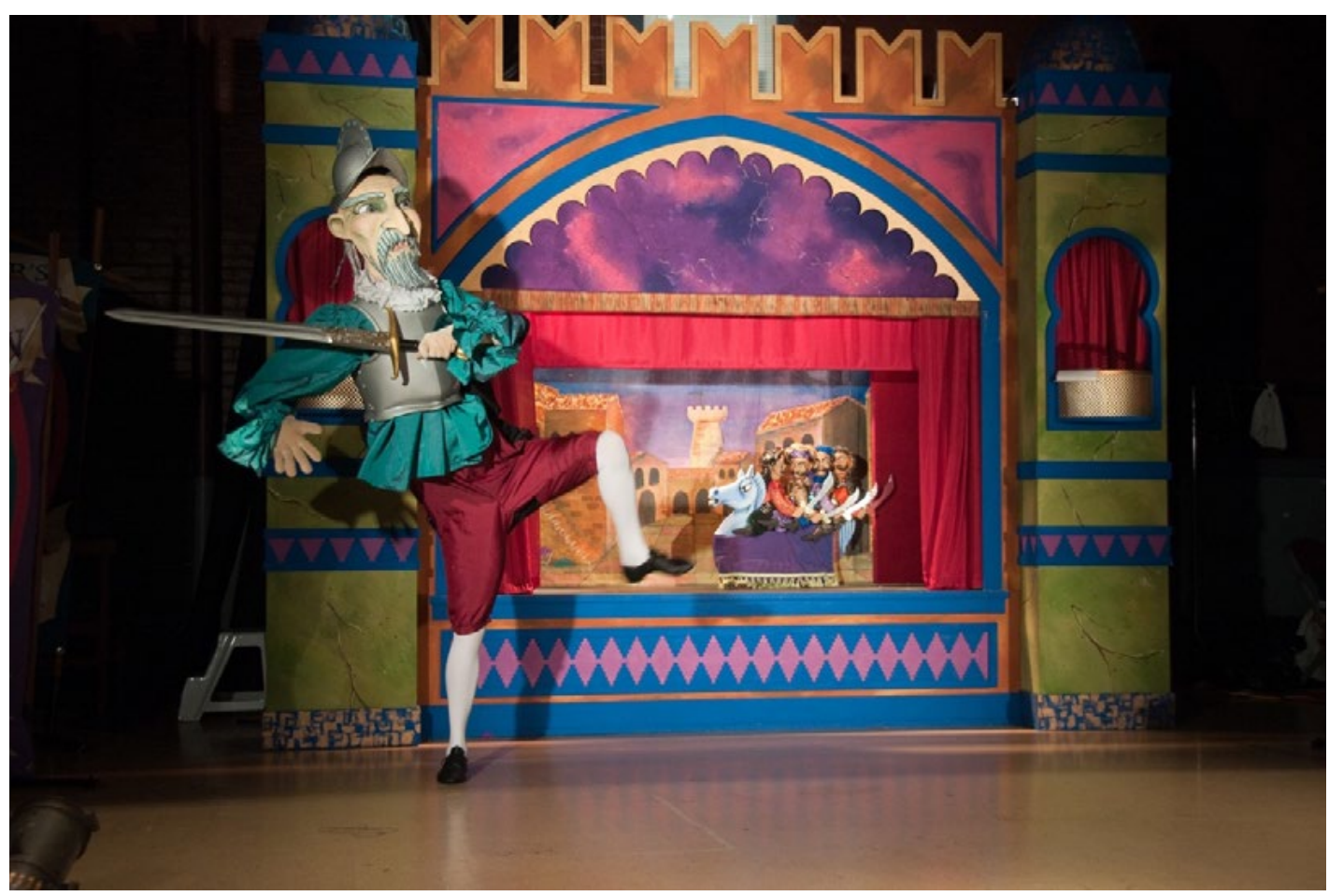

El Retablo de Maese Pedro.

By De Falla, with Cincinnati chamber orchestra et la compagnie Madcap Puppets - USA

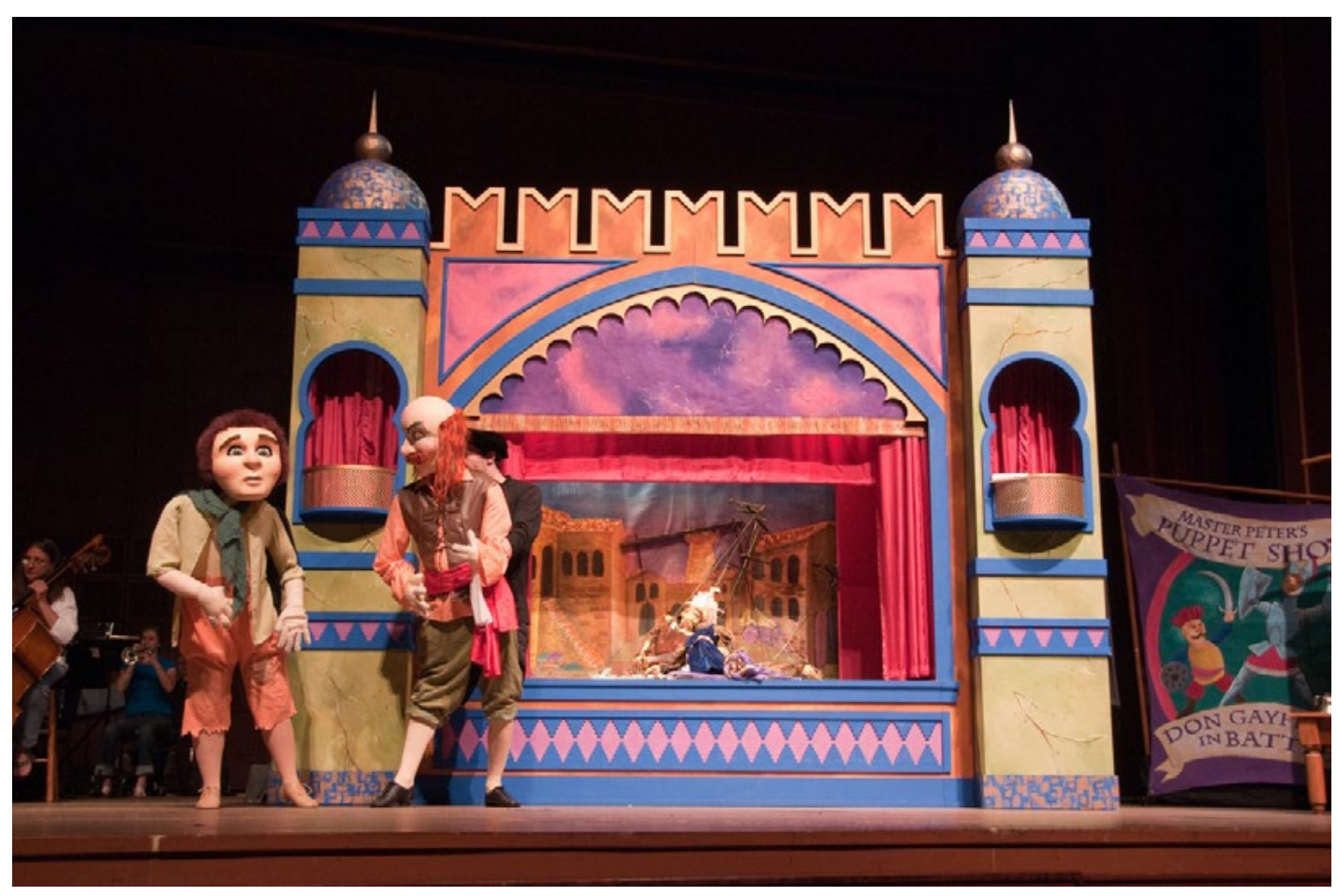

El Retablo de Maese Pedro.

By De Falla, with Cincinnati chamber orchestra et la compagnie Madcap Puppets - USA 


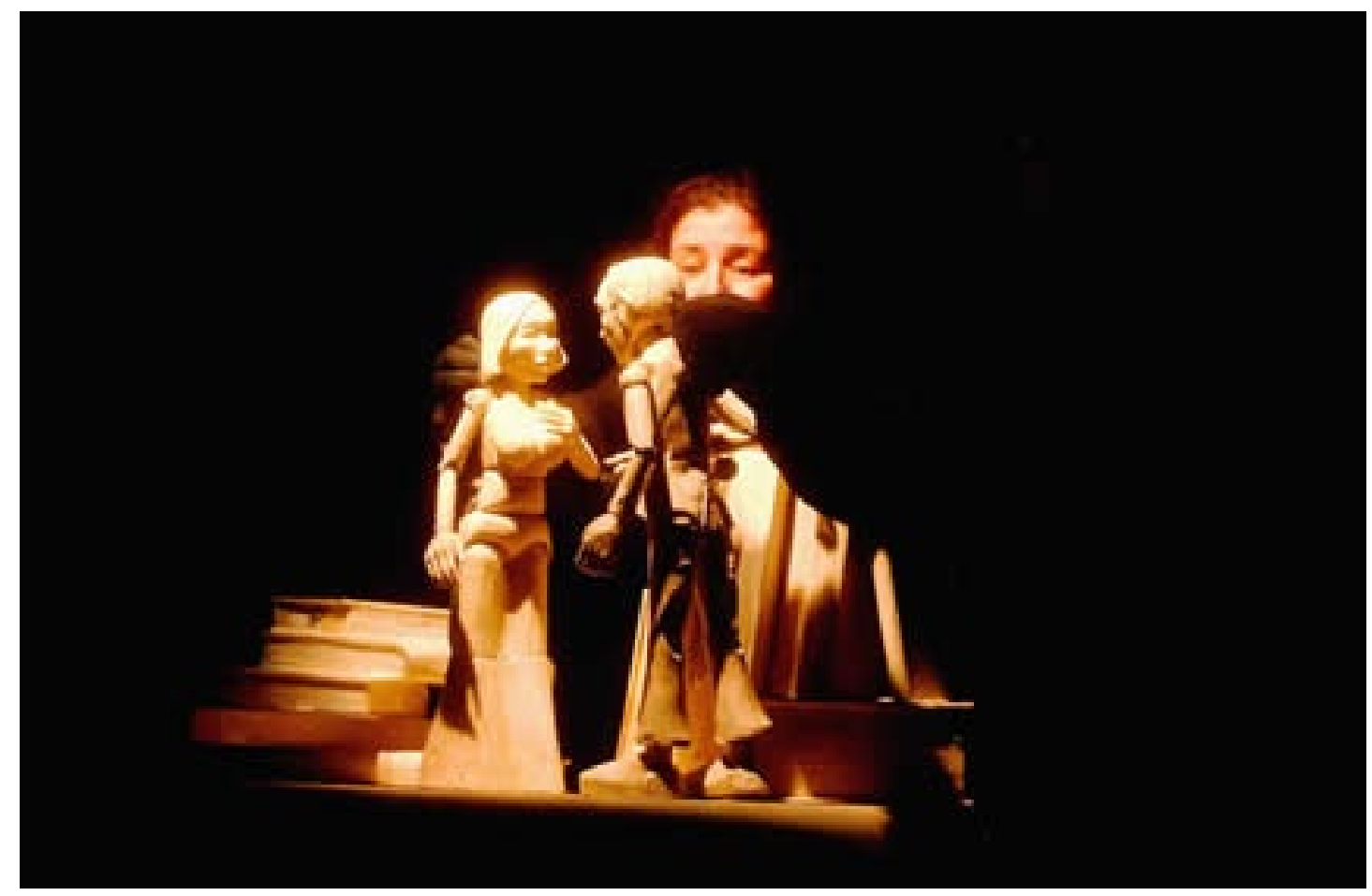

Ariel. Directed: Irina Nicukescu. Texto: Anne Helfgesen. Design e foto: Patrick Maire. Compagnie Lattas. Norway.

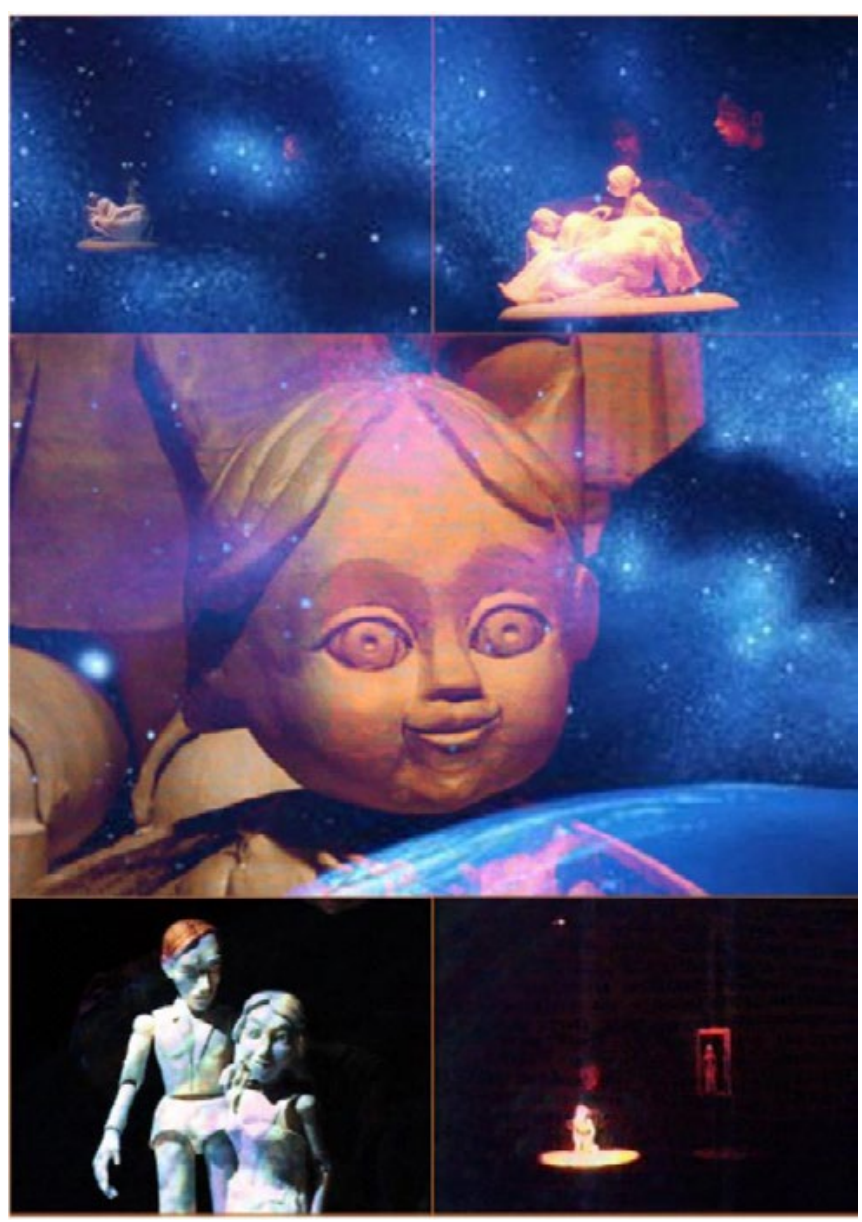

Ariel. Compagnie Lattas. Norvege 


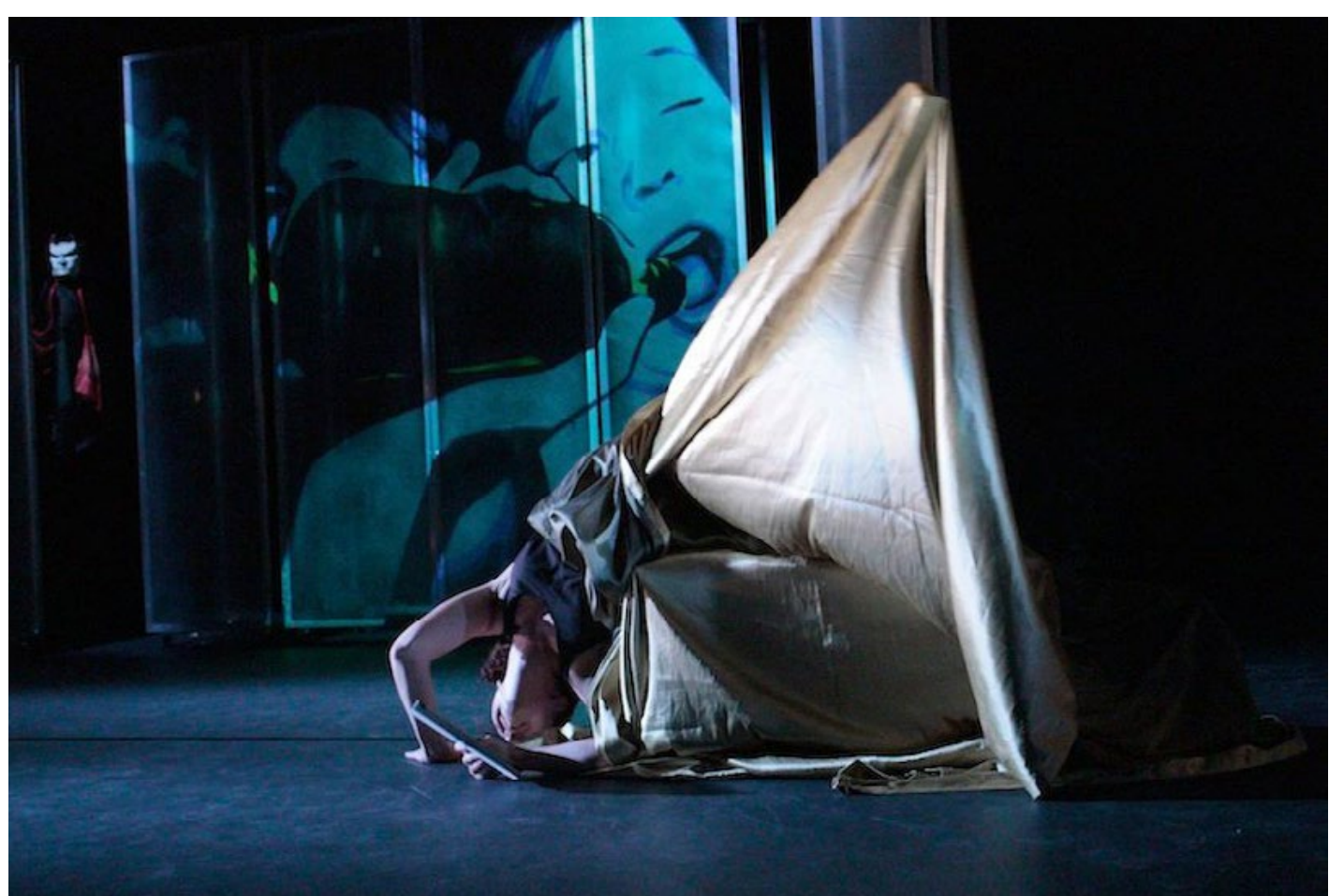

Faustina. Scenario er mise-en-scène Compagnie Lattas. Norvege

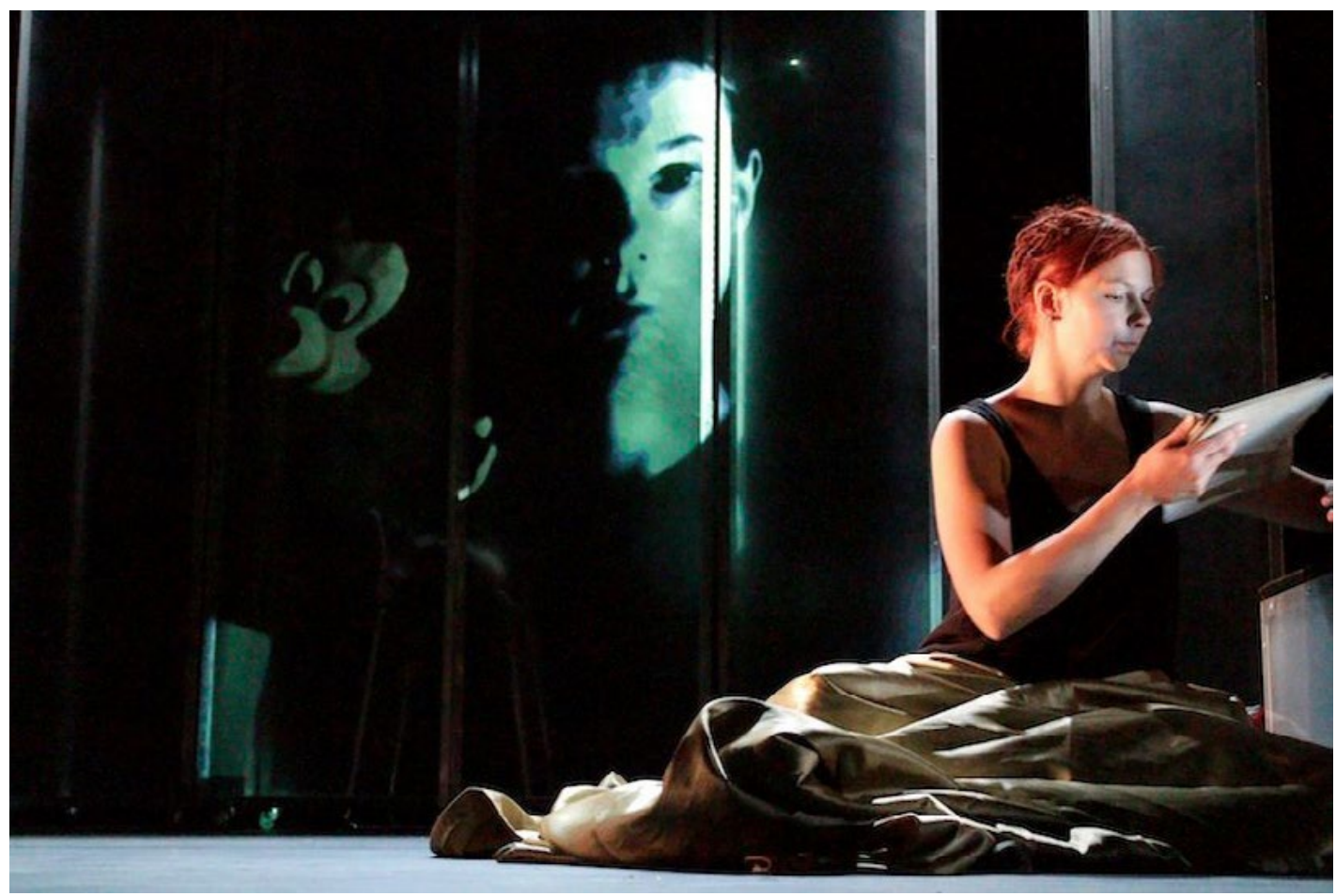

Faustina. Cenário e encenação Companhia Lattas. Noruega 


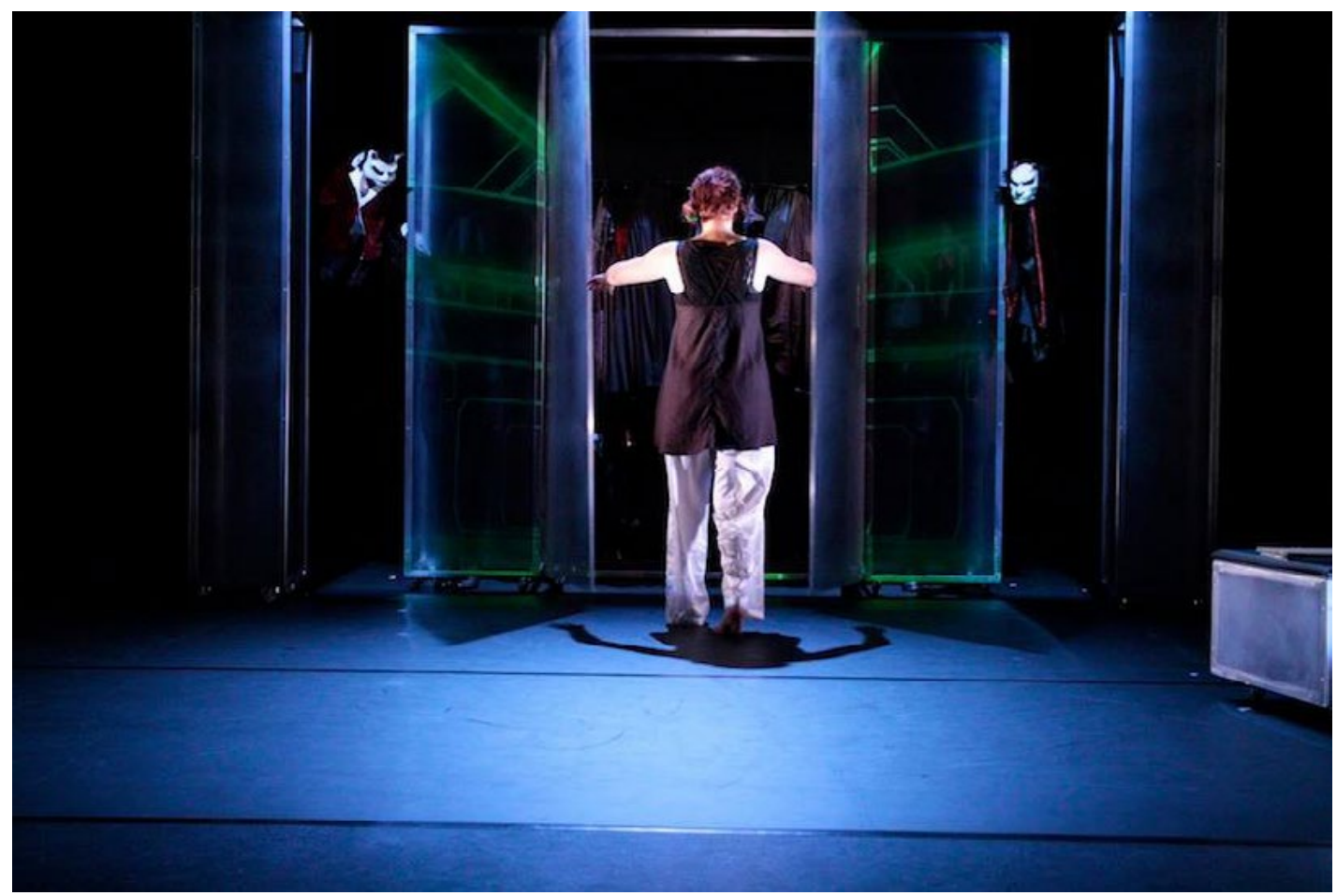

Faustina. Scenario er mise-en-scène Compagnie Lattas. Norvege

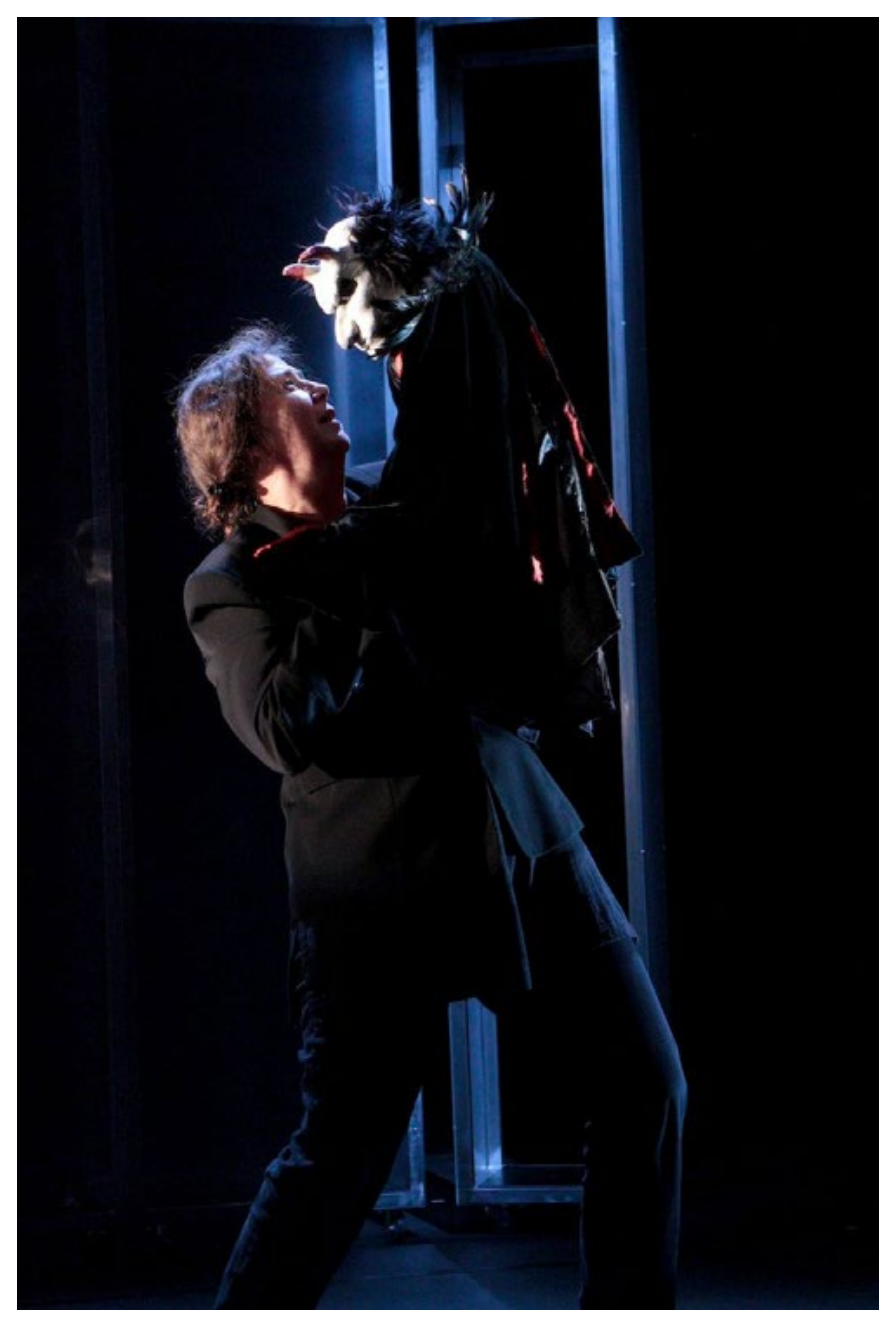




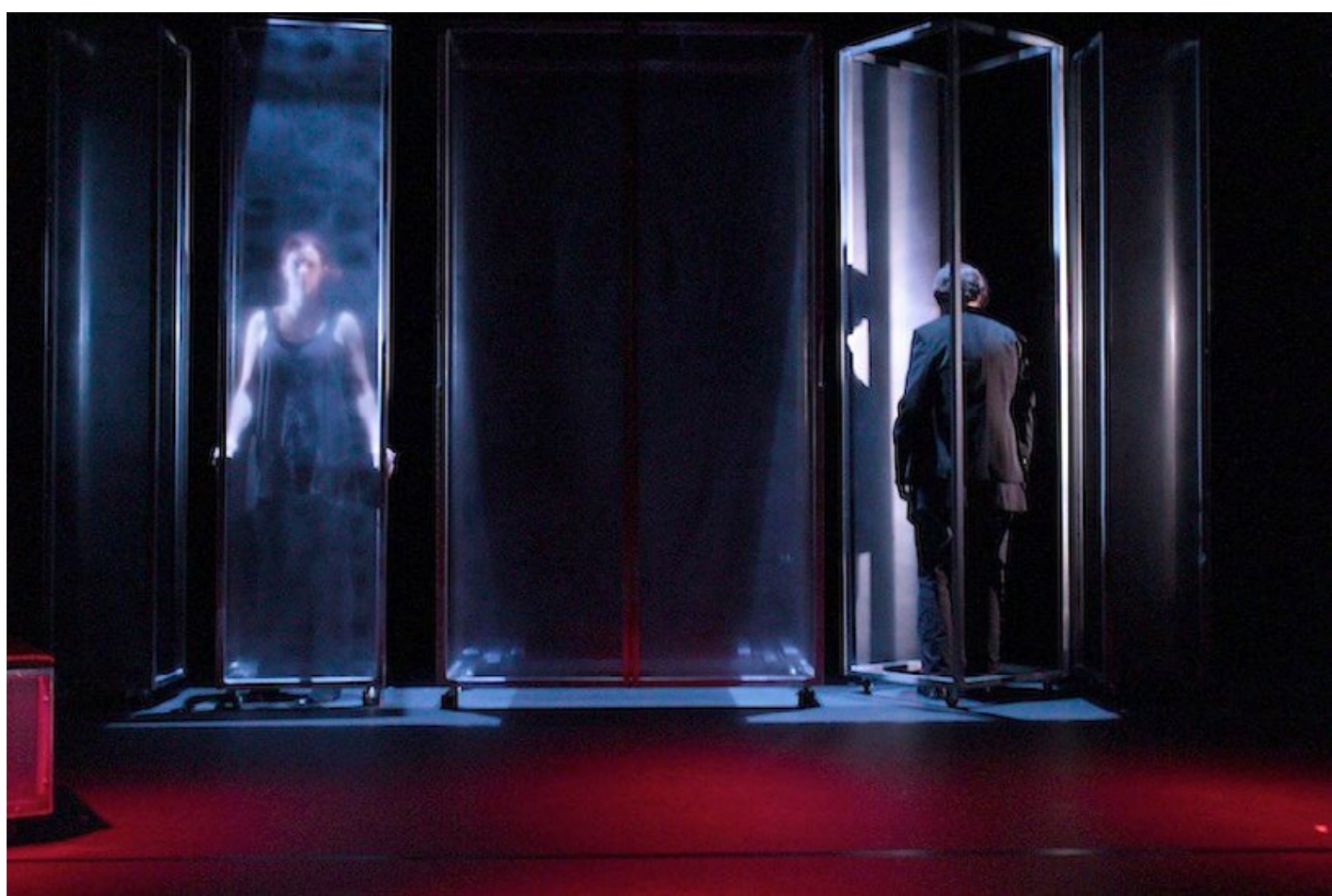

Faustina. Scenario er mise-en-scène Compagnie Lattas. Norvege

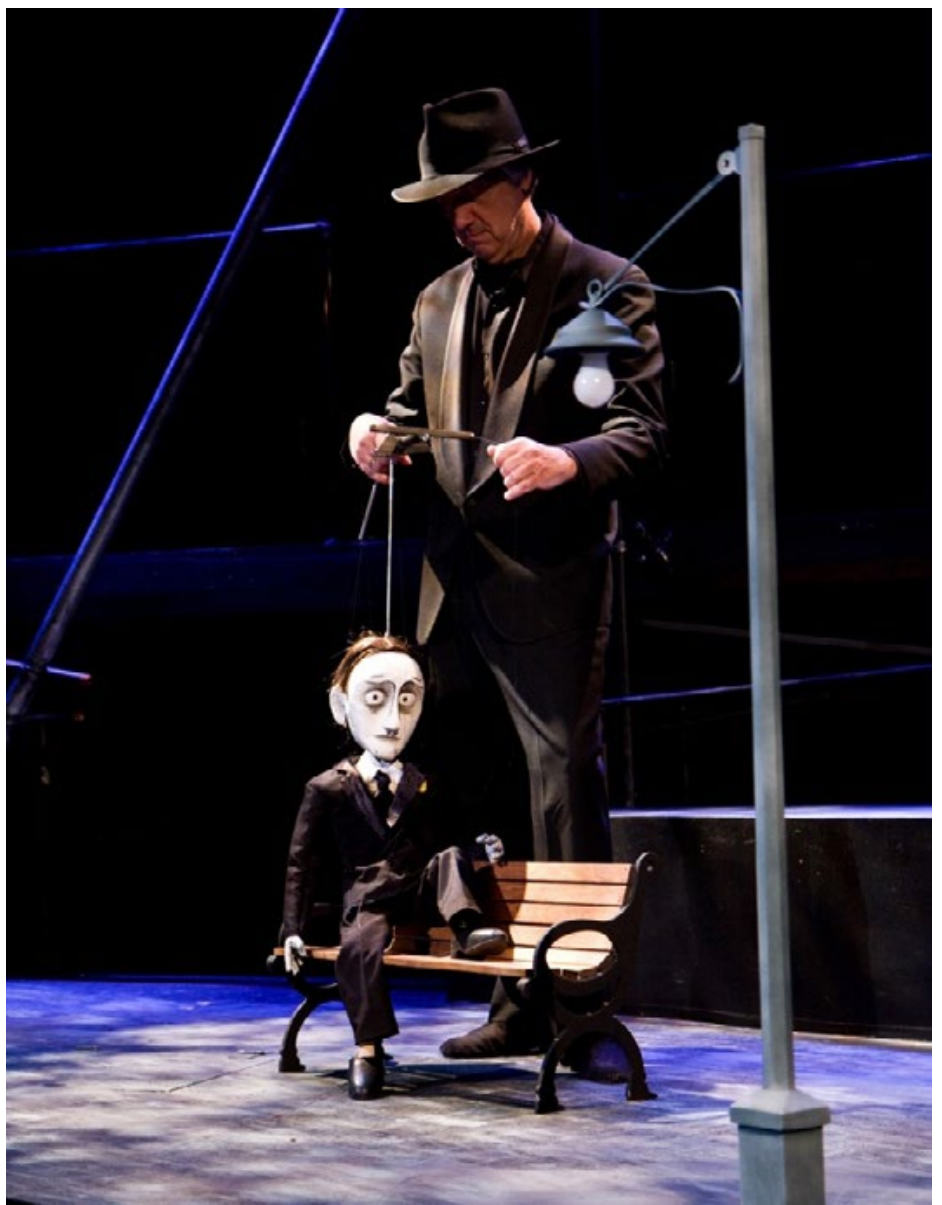

Le Dragon. Know Theatre of Cincinnati and Madcap Puppets. John Lewandowski (Henry). Directing: Irina Niculescu. (2011) - Foto: Deogracias Lerma 


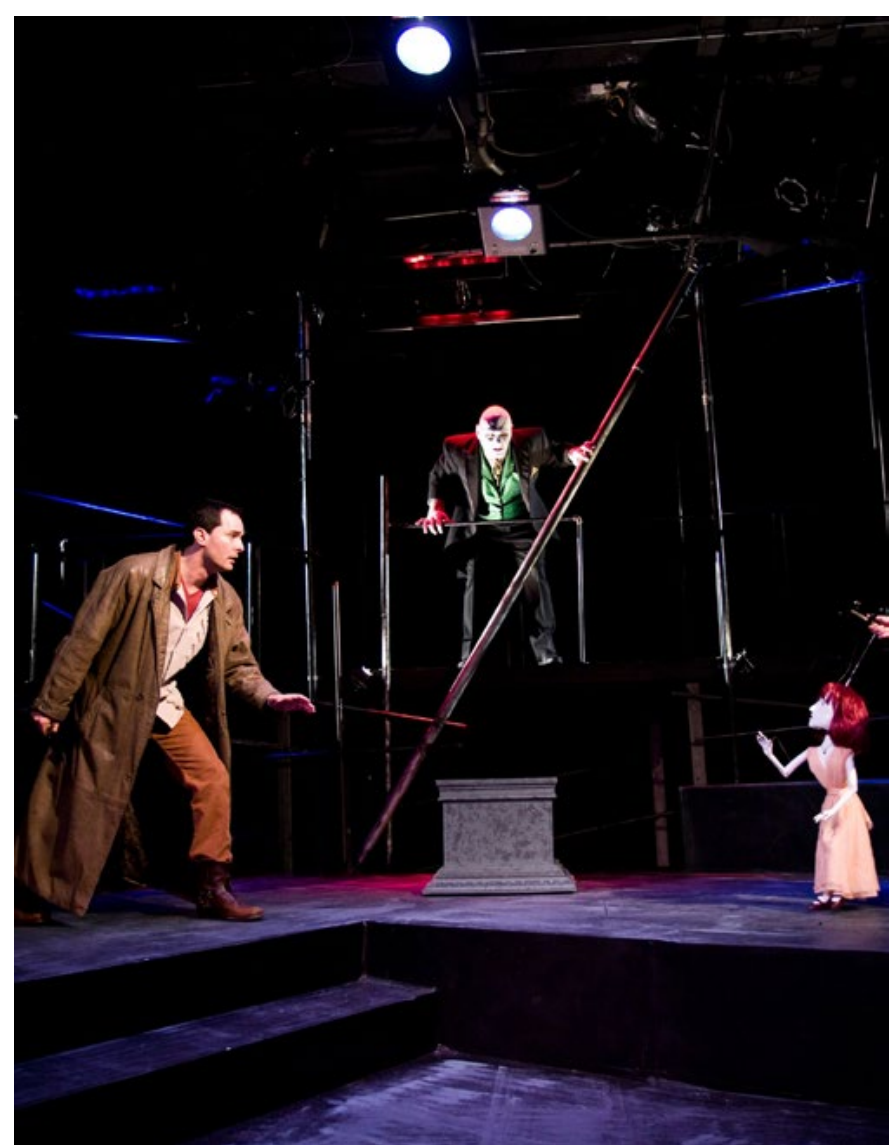

Le Dragon. Tony Bulandra (Lanceot), Elsa and The Dragon. Cincinnati

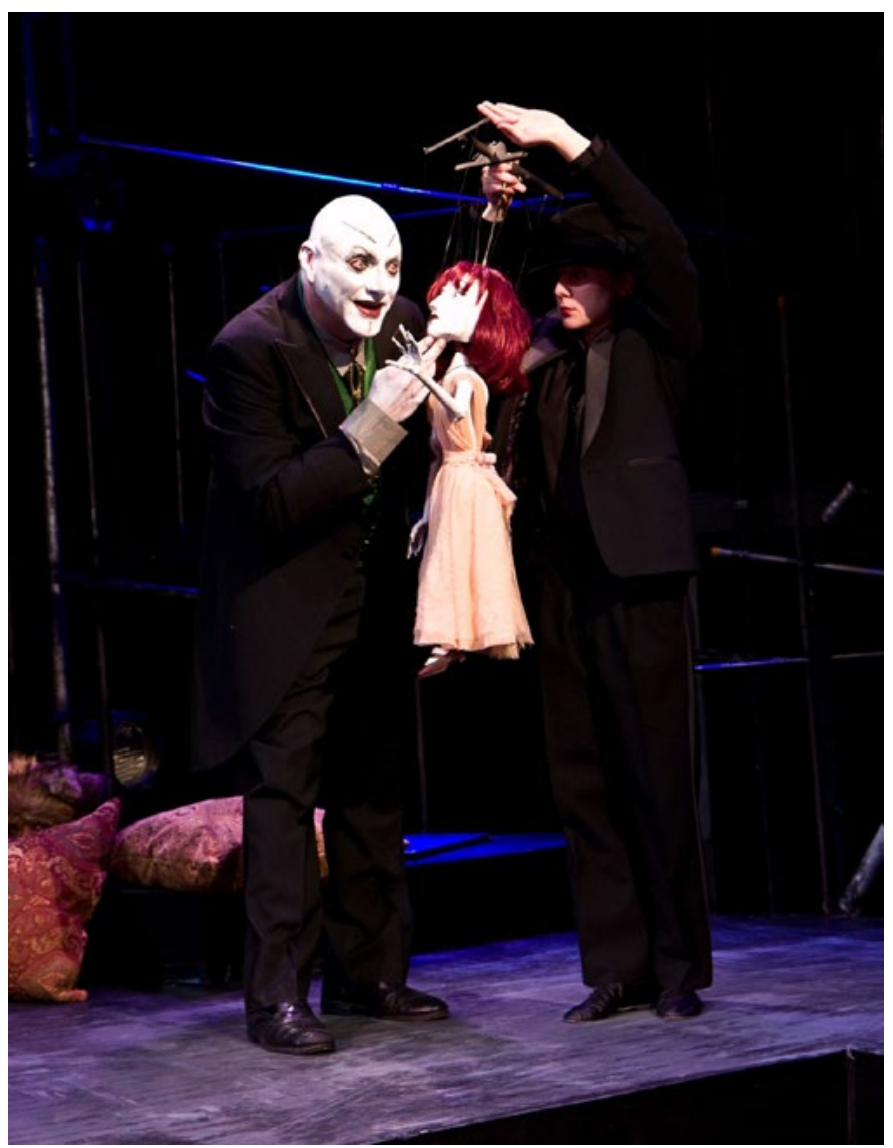

Le Dragon. Know Theatre of Cincinnati and Madcap Puppets. Paul Morris (The Dragon) and Annie Kalahuska (Elsa). Directing: Irina Niculescu. Foto: Deogracias Lerma 


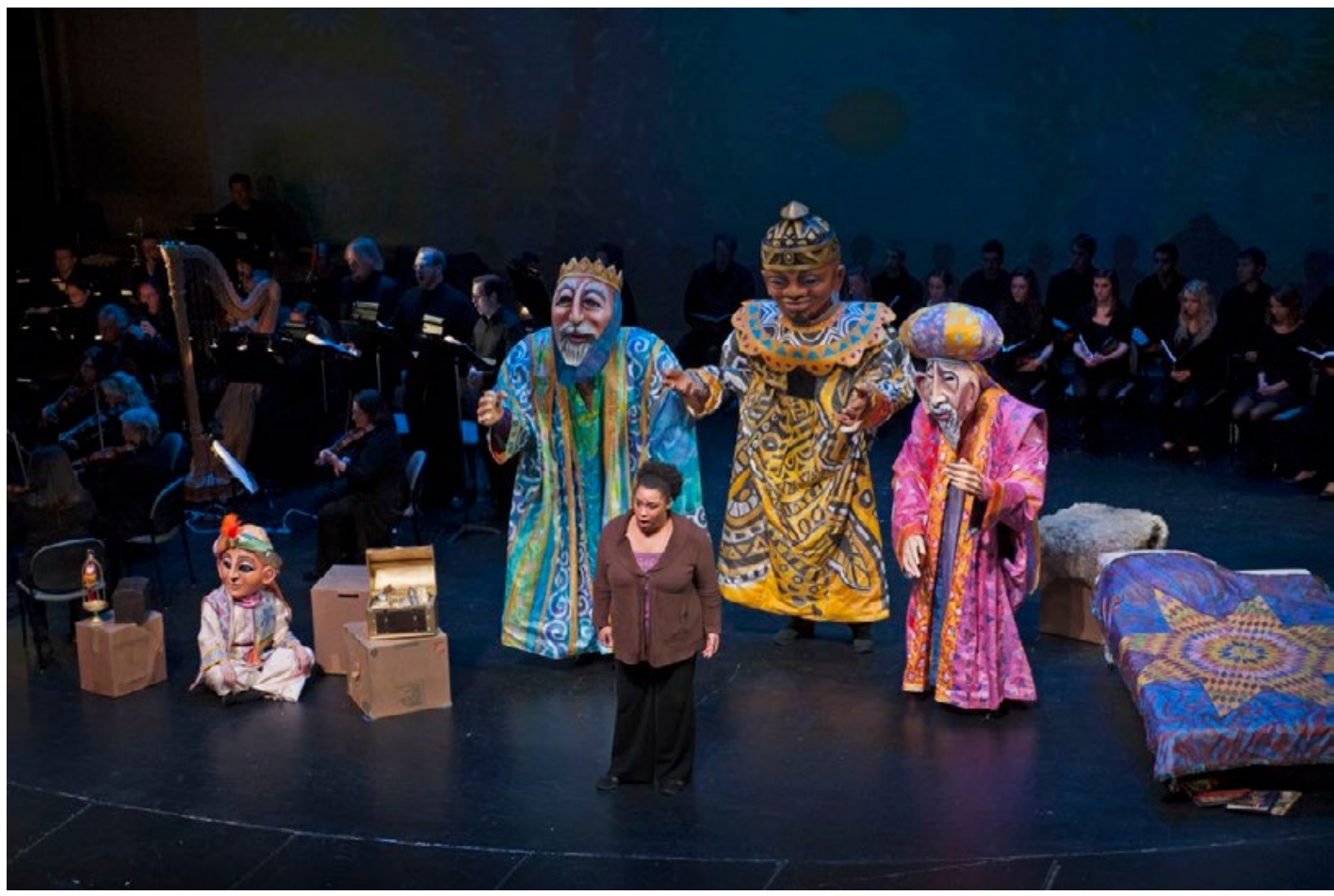

Les Rois Mages. Ópera Amahl and the night visitors. De Gian-Carlo Menotti

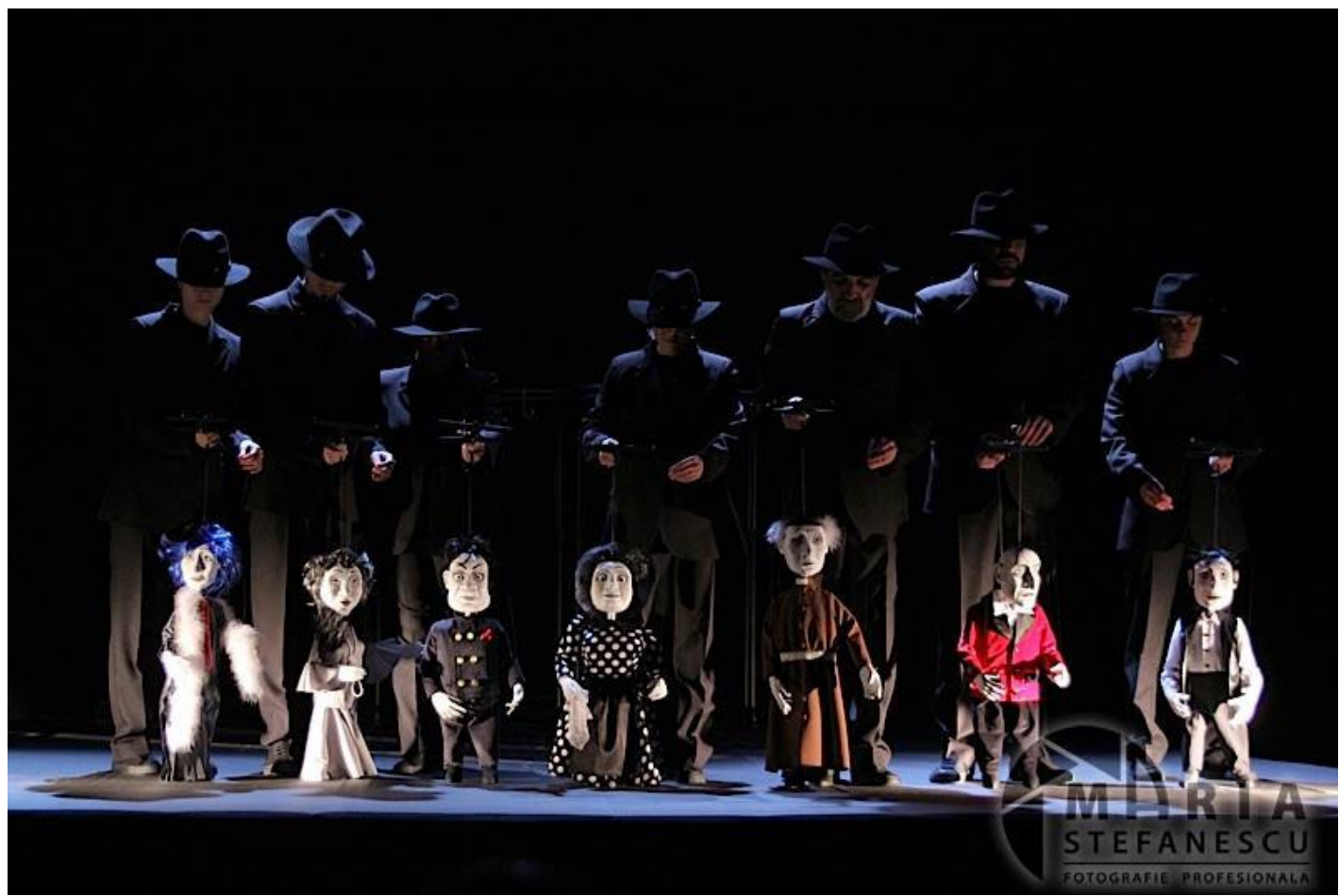

Romeo et Juliette. Prolog, adaptation et mise en scene, Theatre Tony Bulandra, Roumanie 